\title{
CONNAÎTRE PAR L'IMAGE
}

\author{
Jean-Pierre Meunier ${ }^{1}$
}

\section{Introduction}

Quels rapports entre l'image et la connaissance ?

Cette question apparaît quelquefois dans les recherches portant sur les médias, en particulier dans celles qui s'intéressent à leur dimension éducative.

Mais les réponses varient très fort en fonction du cadre théorique de référence et des aspects de l'image et de la connaissance que l'on prend en compte de façon privilégiée. C'est souvent l'opposition image/verbal qui sert de fil conducteur à la réflexion. Or cette opposition peut s'envisager de plusieurs manières. Si l'on évalue l'opposition à travers la dimension proximité/distance par rapport aux choses représentées, l'image sera perçue comme beaucoup plus proche de l'immédiateté (impliquant participation, identification, affectivité, etc.) que le verbal, beaucoup plus propice à la distance et à la réflexion. Si, changeant de point de vue, on aborde l'opposition à travers la dimension sémantique, il sera possible de faire valoir la richesse sémantique de l'image (sa polysémie) contre la limitation des

1 Professeur au Département de communication de l'Université catholique de Louvain. 
mots, et corrolairement, les qualités de la première pour une pédagogie non directive centrée sur les facultés d'interprétation des destinateurs ${ }^{1}$.

Les deux points de vue se défendent du reste très bien car ils correspondent à deux aspects bien réels de la perception des images. Ce qui fait cependant défaut, dans ces recherches, c'est la prise en considération de la connaissance elle-même, telle qu'elle existe en amont et en aval de l'exposition aux images (médiatiques ou autres). De quelle nature sont les représentations mentales qui accueillent les images extérieures et comment celles-ci peuvent-elles s'intégrer à celles-là ?

Le domaine des représentations mentales a été peu pris en compte par les sémiologues. Il s'agit d'un domaine difficile d'accès et souvent considéré comme la propriété des psychologues. Il s'agit aussi d'un domaine dont on pouvait se détourner dès lors que, envisageant les choses de la communication d'un point de vue structuraliste, on considérait les langages comme des réalités autonomes susceptibles d'une description indépendante de toute autre considération que les systèmes de signes eux-mêmes. Cependant, de nouvelles approches sont venues fortement déstabiliser ce point de vue. Depuis le développement de la pragmatique, l'explication de la communication linguistique ne peut plus se passer du recours à l'énonciation et à la relation en contexte (théorie de l'énonciation, théorie austinienne des actes illocutionnaires); ni du recours à la cognition (pragmatique inférentielle, sémantique cognitive). Ces intérêts nouveaux établissent des connexions entre divers domaines (principalement entre la linguistique, la psychosociologie et la psychologie cognitive) et, comme c'est l'usage dans les études de communication, la linguistique entraîne avec elle la sémiotique tout entière dans ces nouvelles relations. Une sémio-pragmatique centrée sur l'énonciation s'est déjà largement constituée tandis qu'on voit apparaître les premiers éléments d'une sémiotique cognitive.

Toujours est-il que, dans cette évolution, la représentation mentale est venue s'immiscer progressivement dans les études de communication comme une nouvelle variable à considérer, une variable qu'il serait même imprudent de ne pas prendre en compte

1 Le premier point de vue énoncé est celui adopté par $D$. Bougnoux dans sa réflexion sur les médias (cf. notamment La communication contre l'information, Paris, Hachette, 1995). Le second est celui adopté par G. Jacquinot dans Image et pédagogie, Paris, PUF, 1977. 
lorsque l'on cherche à comprendre les processus signifiants et les rapports de communication.

Cela étant, il faut tenter de situer l'expérience des images dans l'ensemble des activités cognitives.

\section{Qu'est-ce que connaître?}

Une définition minimale de la connaissance dira sans doute que connaître quelque chose, c'est avoir une représentation de ce quelque chose. Et peut-être ajoutera-t-on -ce qui serait déjà dépasser quelque peu le stade du minimal-que c'est aussi pouvoir manipuler cette représentation pour en tirer des informations.

Il faut alors, si l'on veut avancer, creuser la notion de représentation.

Les psychologues de la cognition reconnaissent deux grandes catégories de représentation : les représentations propositionnelles et les images et, manifestement, c'est en général à la première que vont leurs préférences. Dans un texte de présentation générale des recherches sur les représentations mentales, on peut lire ceci à propos des structures générales de telles représentations :

Au premier rang se trouve la structure propositionnelle. Celleci n'a pas été découverte par des psychologues, mais initialement par des philosophes logiciens : on est redevable de sa conception moderne à l'analyse donnée par Frege (1892). La démarche de la psychologie cognitive a consisté à prendre au sérieux cette analyse, et à formuler d'abord à titre d'hypothèse, l'idée qu'une très grande partie des représentations humaines, et des connaissances correspondantes, est inscrite, coulée, dans le moule propositionnel.

Formuler cette hypothèse, c'est supposer que les représentations humaines sont organisées, pour l'essentiel, comme l'est le langage. Cela ne veut pas dire, comme on le croit parfois, que la cognition serait calquée sur le langage, dérivée de lui, ou toujours exprimable. Cela signifie au contraire, que la structure du langage est dépendante de celle des représentations cognitives, que si le langage est comme il est, c'est parce que la cognition lui préexiste ${ }^{1}$.

1 «Les représentations mentales » in J.-F. LE Ny et M. D. GINESTA (sous la dir. de), La psychologie, Paris, Larousse, coll. "Textes essentiels", 1995, p. 270. 
Par opposition aux représentations propositionnelles, les représentations imagées sont caractérisées comme ayant un rapport analogique avec la perception. Leur existence n'est pas contestée mais leur importance est minimisée au point que certains auteurs pensent pouvoir la réduire à la structure propositionnelle.

Outre sa clarté sur la position de nombreux psychologues concernant la représentation, le texte cité plus haut présente encore l'intérêt de laisser transparaître certaines des influences qui ont pu les inspirer. Celle de la logique dont les développements ont pesé sur notre conception du fonctionnement de l'esprit. Celle, aussi, d'un certain logocentrisme, car, on peut penser que la prééminence de la structure propositionnelle soit le résultat de la projection sur le domaine mental de la hiérarchie culturellement établie-surtout avec l'écriture et le livre- entre le langage verbal et l'image. Ce ne serait pas la première fois qu'un produit dérivé serait mis à la source.

Cependant, contre cette tendance lourde en faveur de la représentation propositionnelle, il est un certain nombre de travaux qui tendent à restituer à l'image la prééminence -voire l'exclusive-dans l'ordre des représentations mentales. Et curieusement, certains des principaux arguments allant dans ce sens proviennent de linguistes.

J'ai tenté de montrer ailleurs comment ces recherches ont permis à l'analogique de s'immiscer progressivement dans la zone réservée au digital ${ }^{1}$. Je ne ferai ici que rappeler rapidement cette progression.

On se souvient que Piaget faisait émerger le concept verbal de l'imagerie mentale mais reconnaissait néanmoins à celle-ci une certaine fonction d'illustration du signifié conceptuel -conçu comme classe abstraite.

Le signifiant de ce schéma (la classe générale) ne serait autre que le mot ou signe verbal, et l'imagerie imitative ne servirait alors que de symbole individuel doublant à titre d'adjuvant intérieur, le signe collectif: l'image devenant ainsi bien distincte du concept ${ }^{2}$.

La séparation entre l'image et le concept, déjà fragilisée par ce rôle d'illustration maintenu pour la première, l'a été davantage encore

1 Cf. J.-P. MEunier, "Y a-t-il de l'image dans les verbes ? Pour une reformulation des rapports entre l'analogique et le digital", MEI, «Médiation et information», $\mathrm{n}^{\circ}$ 6, Icône-image, 1997, pp. 113-124. 2 J. PIAGET, La formation du symbole chez l'enfant, Neuchâtel, Delachaux et Niestlé,
1976 (1945), p. 160. 
par les recherches expérimentales montrant l'existence d'une composante imagée dans le concept lexical -en termes saussuriens : dans le signifié du signe verbal. Le concept de "valeur d'imagerie des mots" rend compte de la capacité qu'ils ont de susciter la formation d'image mentale ${ }^{1}$. Cet aspect cependant n'est pas, du point de vue même de ceux qui effectuent des recherches à son sujet, essentiel, puisqu'il coexiste avec d'autres, abstraits, qui ne doivent rien à l'image.

La théorie des modèles mentaux, dont un des principaux représentants est sans doute le psychologue Ph. Johnson-Laird, permet un pas de plus vers la reconnaissance du caractère essentiel de l'image. Dans le cadre de cette théorie, les énoncés verbaux sont l'objet d'un traitement qui les transforme en un modèle mental, une sorte d'analogue de la situation décrite dans le discours.

Les humains perçoivent le monde et en construisent des modèles (...). Ils peuvent aussi reproduire ces modèles dans le discours, c'est-à-dire produire des comportements symboliques -des expressions linguistiques-censés transmettre les modèles à quelqu'un d'autre. De son côté, l'individu qui décode ces expressions linguistiques construit un modèle qui ressemble à l'état du monde que le locuteur a connu et voulu transmettre ${ }^{2}$.

La communication verbo-digitale perd ainsi toute préséance car elle ne semble avoir d'autre finalité que la représentation analogique qu'elle permet de construire.

C'est encore plus évident dans le cadre de la "sémantique cognitive" qui, après avoir clairement remis en cause le postulat (structuraliste) de l'autonomie du langage (donc de la dépendance du signifié par rapport au signifiant et à sa logique), a entrepris l'exploration des rapports entre le langage et les structures conceptuelles et mis au jour dans celles-ci le rôle fondamental de l'imagerie.

Pour R. Langacker, par exemple, le concept d'image est nécessaire pour rendre compte du fait que différentes expressions linguistiques donnent des vues variées d'une même situation objective ${ }^{3}$. Ces vues diffèrent selon les aspects de la situation qu'elles sélectionnent, le degré de saillance de ces aspects, leur degré d'abstraction, la

1 Cf. M. DENIS, Image et cognition, Paris, P.U.F., 1989.

$2 \mathrm{Ph}$. JOHNSON-LAIRD, L'ordinateur et l'esprit, Paris, Éd. Odile Jacob, 1994.

3 Cf. R. LANGaCKER, Foundations of Cognitive Grammar, vol. 1, Stanford (California), Stanford University Press, 1987. 
perspective sous laquelle ils sont vus, etc. (on y reviendra plus loin). Les conventions lexicales et grammaticales sont là précisément pour fournir les expressions nécessaires à cette structuration!

Loin de constituer une structure autonome dont les combinaisons ne dépendraient que de règles formelles, la grammaire dépendrait donc du sens, c'est-à-dire de l'imagerie.

Il semble donc y avoir quelque chose de "chosiforme" à tous les niveaux de la communication verbale. A moins qu'elle ne se réduise à un acte purement phatique -ou "expressif" dans la terminologie de la théorie des actes illocutionnaires- une énonciation verbale est communication d'un modèle mental à caractère iconique et la langue (lexique et grammaire) est fondamentalement un ensemble d'instruments au service de la composition analogique.

Tout cela plaide en faveur de l'iconicité de la pensée. Connaître quelque chose -une chose, une personne, un processus, une situation...- c'est avoir de ce quelque chose une représentation iconique : une image plus ou moins précise et plus ou moins multisensorielle ou, aux niveaux supérieurs de complexité, un modèle plus ou moins abstrait. Les problèmes que pose cette modélisation sont nombreux : quels sont les degrés et niveaux d'abstraction des modèles ? Quelles sont les opérations cognitives qu'autorisent les différentes formes et degrés d'iconicité ? Comment s'organisent entre eux ces modèles ? Etc.

Ces questions intéressent avant tout les psychologues qui, du reste, ont déjà donné de nombreux éléments de réponse à ces questions ${ }^{2}$.

Mais elles intéressent aussi, évidemment les linguistes et psycholinguistes ainsi que les sémiologues et chercheurs en communication, d'une manière qui demande qu'on s'y arrête quelque peu.

1 Un exemple simple pour illustrer cette pensée très complexe. Les deux phrases suivantes: He sent a letter to Susan.

He sent Susan a letter.

qui réfèrent à une même situation objective, diffèrent néanmoins par les images qu'elles induisent : alors que la première rend saillant le chemin parcouru par la lettre, la seconde rend saillant l'état final -la possession de la lettre par Susan.

2 On trouvera un aperçu panoramique des recherches sur les modèles mentaux dans M.-F. EHRLICH (coord.), Les modèles mentaux, Paris, Masson, 1993. 


\section{Indice, icône, symbole}

Les problèmes des sémiologues et théoriciens de la communication sont proches mais néanmoins sensiblement différents de ceux que rencontrent les linguistes. Ceux-ci n'ont à prendre en considération que la langue. Ceux-là doivent élargir leur champ d'investigation pour y inclure toutes les sortes de signes : les objets porteurs de signification, les images dans leur grande diversité (fixes, animées, artistiques, médiatiques, etc.), les gestes, les sons, etc., etc. Cet élargissement, du reste, prend souvent pour point de départ le travail déjà effectué par le linguiste à propos de la langue, ce qui ne va pas sans poser quelques sérieux problèmes d'extrapolation.

Mais ce sont précisément ces problèmes qui rendent saillants, aux yeux des sémiologues, des aspects et questions qui échappent facilement aux linguistes. La prise en considération exclusive d'un seul système de signes peut le faire apparaître comme un médium relativement neutre, un simple moyen de communiquer indifférent à cela même qui est communiqué. Au contraire, la nécessaire prise en compte de la diversité des signes fait apparaître leur spécificité. On se souvient comment l'élargissement à l'image des notions structuralistes (signifiant, signifié, syntagme, paradigme) a rendu évidents, à la fois, la relative inadéquation de ces notions et certains caractères propres à la signification iconique. Il en fut de même avec l'extrapolation au domaine iconique des notions pragmatiques (énonciation, actes illocutoires, ...); il en est sorti une sémio-pragmatique qui s'est écartée de son patron linguistique au fur et à mesure qu'elle devait s'adapter aux caractéristiques de l'image.

La confrontation avec la dure spécificité des signes a induit une problématique -propre à mon avis au sémiologue et au théoricien de la communication- où se trouvent soulevées des questions relatives à cette spécificité : la question des niveaux de signification par exemple (qui interfère, chez Bateson et Watzlawick, avec la différenciation analogique-digital), ou la question des sémantiques et syntaxes respectives.

Cette problématique explique le recours de plus en plus fréquent à la sémiotique de Peirce, au moins à la fameuse trichotomie indiceicône-symbole. C'est que cette triade offre de multiples avantages. Tout d'abord, elle prend immédiatement en compte la variété et la spécificité des signes tout en donnant le moyen de les catégoriser (donc d'échapper au singulier). Ensuite et surtout, elle implique l'idée 
d'une hiérarchie de niveaux et celle de passage d'un niveau à un autre. Or, cette représentation hiérarchique -assez abstraite et théorique dans la sémiotique de Peirce lui-même-présente le grand intérêt d'entrer en résonance avec les représentations hiérarchisées élaborées en psychologie (les processus primaire et secondaire chez Freud, l'imaginaire et le symbolique dans la psychanalyse lacanienne), en psychosociologie (l'analogique et le digital) ou encore en psychologie du développement. Dans ce dernier domaine, il est sans doute utile de rappeler -ce que beaucoup de sémiologues ignorentque le grand psychologue du développement qu'était Piaget utilisait directement les catégories de Peirce -indice, icône, symbole- pour décrire le développement par étapes de la "fonction symbolique"1. Ces mises en correspondance ont permis l'élucidation de nombreux rapports entre les grandes catégories de signes et les différents aspects de la communication dégagés par les théories successives de la communication : la signification, la relation et la cognition.

Sur le plan de la relation, la correspondance établie entre les catégories peircéennes et les couples principe de plaisir/principe de réalité et, surtout, imaginaire/symbolique, met en évidence une progression ontogénétique entre d'une part, l'indiciel et l'iconique qui apparaissent liés au contact, à la relation fusionnelle ou communautaire, et le symbolique, lié au langage, qui apparaît comme un opérateur de distanciation dans la relation moi-autrui-le monde ${ }^{2}$.

Sur les plans de la signification et de la cognition -plans inséparables dans une large mesure- la hiérarchie indice-icône-symbole permet de même de concevoir une progression entre d'une part les processus que l'on peut qualifier de primaires ou prélogiques (condensation et déplacement ou métaphore et métonymie) et d'autre part les processus logico-langagiers. Le cadre conceptuel constitué par la triade indice-icône-symbole a ainsi montré une certaine fécondité. Il a en tout cas permis d'établir une chose que la linguistique ignore ou néglige : c'est que la communication varie considérablement avec

1 Cf. à ce sujet J. PIAGET, La formation du symbole chez l'enfant, op. cit. Nous avons, D. PERAYA et moi-même largement exploité la théorie piagétienne pour caractériser les différents types de signes; cf. Introduction aux théories de la communication, Bruxelles, De Boeck Université, 1993.

2 D. Bougnoux a bien mis en évidence cette progression, notamment dans $L a$ communication par la bande, Paris, La Découverte, 1991 et La communication contre l'information, op. cit. 
les types de signes qui y sont impliqués et ceci sous quelque aspect qu'on l'envisage : relation, signification, ou cognition.

Il faut cependant admettre que les théories cognitives du langage évoquées plus haut posent un sérieux problème à la triade peircéenne.

La progressive "iconicisation" de la pensée perturbe en effet les correspondances établies. Si l'imagerie déborde de son domaine -la représentation iconique proprement dite, impliquant images mentales et images matérielles- pour envahir la communication linguistique, on ne voit plus bien comment maintenir les caractéristiques spécifiques de l'une et de l'autre. Une solution consisterait à dissoudre les différences et à rabattre le linguistique (digital, voué à la logique) sur l'iconique, un peu comme la sémiologie structuraliste avait tenté l'opération inverse. Le linguistique ne serait plus alors que l'instrument de l'iconique. Mais, malgré tout, l'intuition subsiste que la distinction a un sens et qu'il existe de bonnes raisons de la maintenir, ne fût-ce que sur le plan pragmatique de la relation au monde et à autrui où il semble évident et acquis -au moins depuis l'observation de Freud du jeu du fort-da- que le langage est indispensable à l'établissement des distances et distinctions (je-tu-il). Plutôt qu'à une simplification du problème par mise en valeur exclusive d'une dimension (la dimension iconique), c'est à une complexification que l'on est convié.

L'évitement de l'unidimensionnalité conduit alors à de difficiles problèmes d'interaction ou de médiation. Si l'on admet la finalité iconique du linguistique, il faut néanmoins comprendre les effets de la médiation exercée par le deuxième. Le signifié, en tant qu'imagerie, ne peut se résorber dans le signifiant et devenir pur concept mais le signifiant, avec son arbitrarité et sa négativité, doit intervenir dans la structuration de l'imagerie. Ainsi peut-on supposer que c'est à travers les mots que les images se construisent en classes centrées sur des exemples prototypiques et que sont rendues possibles certaines opérations mentales -comme la déduction. Mais une telle structuration et de telles opérations doivent être repensées à partir de leur substrat iconique. De son côté, ce dernier ne peut plus se comprendre au moyen de l'un ou l'autre exemple simple (l'image mentale d'un objet ou d'une personne, par exemple, ou sa photographie) car dès le moment où l'on considère son imbrication avec le langage, on ne peut plus le concevoir que complexe, comportant des niveaux d'abstraction et servant de support à des opérations cognitives diverses, y compris les opérations logiques qui apparaissaient comme relevant du langage 
en tant que tel (que celui-ci soit conçu comme langage au sens propre -linguistique- ou, à la manière des psychologues, comme langage de la pensée).

La reconnaissance d'une imagerie mentale complexe fait alors surgir -ou plutôt rend plus saillant- un nouveau problème : celui des rapports qu'elle entretient avec les images matérielles : dessin, photographie, cinéma, vidéo, cartes géographiques, plans, graphiques, schémas, signaux routiers, icônes des ordinateurs... Tant qu'elles étaient considérées comme parties de codes analogiques relativement autonomes ou comme illustrations du discours, ces images pouvaient être étudiées pour elles-mêmes. Mais, dès lors qu'elles s'insèrent dans une pensée iconique complexe, il faut comprendre les modalités de cette insertion.

On voit ainsi se dessiner le programme d'une sémiotique cognitive: il s'agit de spécifier les différentes dimensions de l'imagerie mentale et d'examiner la manière dont elle interagit avec les divers systèmes sémiotiques construits par la culture et la technique.

On tentera, dans les lignes qui suivent, de mieux cerner certaines des questions qui viennent d'être évoquées. On s'appuiera pour cela sur divers travaux de sciences cognitives. Mais c'est surtout l'approche intuitive -introspective- qui dominera. Aussi, qu'il soit bien clair qu'il ne s'agira que d'hypothèses et de suggestions, une simple recherche exploratoire de phénomènes encore peu pris en considération dans les études de communication.

\section{Les images originelles (ou le mimétisme à l'origine des images)}

L'image, pensait Piaget, s'enracine dans l'imitation. Cette hypothèse est ignorée dans les recherches d'inspiration cognitiviste. Elle mérite pourtant d'être considérée avec la plus grande attention car le processus qu'elle décrit contient en germe tous les développements cognitifs qui suivront. L'imitation est d'abord imitation en acte : face à un autre ou à un objet, l'enfant manifeste une capacité de reproduction mimétique de cet autre ou de cet objet. Peu importe ici l'âge auquel commencent ces jeux de miroir -plus tôt semble-t-il que ne le pensait Piaget lui-même-, l'important ici étant de reconnaître qu'ils constituent une forme primitive ou primordiale de connaissance. Du 
reste, dans la perspective piagétienne, l'imitation est considérée comme accommodation à l'objet. Un exemple pour illustrer ce point :

Laurent regarde une boîte d'allumettes que je tiens dressée dans le sens de la hauteur et que j'ouvre et ferme alternativement. Enchanté de ce spectacle qu'il regarde avec la plus grande attention, il imite la boîte de trois manières. $1^{\circ}$ Il ouvre et ferme sa main droite tout en regardant l'objet. $2^{\circ} \mathrm{Il}$ fait $\mathrm{Tff}$ Tff avec la bouche pour reproduire le bruit de l'objet. $3^{\circ}$ Enfin (...) il ouvre et ferme la bouche $(. . .)^{1}$.

Par la suite, l'imitation devient différée puis intériorisée, c'est-àdire réduite à des esquisses ou ébauches motrices qui serviront de base à l'élaboration d'image mentale, au jeu et au dessin.

Ce court rappel de la perspective piagétienne pour étayer quelques idées qui serviront de base pour la suite :

- la connaissance s'enracine dans l'imitation ou (je préfère le terme) dans la mimésis comme capacité primitive de reproduction corporelle (posturale, motrice) du monde perçu. La représentation s'origine dans cette faculté reproductrice.

- la mimésis est un phénomène complexe comportant une sorte de vicariance des différentes parties du corps; comme le montre l'exemple de la boîte d'allumettes, l'enfant imitant reproduit avec différents organes (la main, la bouche) qui se font écho et se complètent mutuellement.

— le mimétisme est autant à la réception qu'à la production des images; percevoir une image implique inévitablement une reprise posturale et motrice des formes perçues.

Cette dernière affirmation peut, selon moi, être généralisée à des représentations plus complexes et/ou plus abstraites que celles envisagées par Piaget. Lorsqu'une image -mentale ou matérielle-comporte des scènes avec des personnages, sa réception comme sa production suppose des processus de projection-identifications multiples portant sur tous les personnages. Quant aux modèles mentaux -dont il va être bientôt question-, mêmes réduits à quelques entités abstraites en interaction, on peut raisonnablement supposer qu'ils induisent des processus similaires. Cette idée s'accorde avec les vues de plusieurs des théoriciens des modèles mentaux.

1 J. PIAGET, La formation du symbole chez l'enfant, op. cit., p. 66. 
Denis et Vega, des psychologues expérimentaux qu'on ne peut soupçonner de se laisser aller à des impressions subjectives, écrivent :

La seconde différenciation importante entre images et modèles est que si les chercheurs se sont intéressés aux modèles visuospatiaux, les modèles mentaux peuvent également représenter des entités et des relations de nature non spatiale. Ainsi lorsqu'un lecteur prend connaissance de la description d'un personnage, il est vraisemblable qu'il en construit une représentation qui n'est pas seulement celle d'une entité physique autour de laquelle est bâti un scénario, mais la représentation d'un riche ensemble d'informations psychosociales à propos du personnage. Aux personnages sont attachés des buts, des rôles, des intentions, des états émotionnels ${ }^{1}$.

Mais d'où viennent les buts, rôles, intentions, états émotionnels? Sinon des projections-identifications qui lient le récepteur aux images.

Les modèles mentaux, écrit par ailleurs P. Lévy, ne sont pas liés à une seule modalité perceptive. On peut leur donner des traductions visuelles, mais aussi haptiques, proprioceptives, ou même leur trouver des équivalents sous forme d'enchaînement de schémas moteurs ${ }^{2}$.

Les entités d'un modèle abstrait (taches visuelles, zones plus ou moins délimitées $d u$ fond...) tirent leur caractère statique ou dynamique de la façon dont le corps (via le regard) les investit en leur imprimant des mouvements, en les figeant dans des positions, en projetant sur eux des intentions.

On peut donc supposer une continuité entre les premières imitations et les modèles mentaux les plus abstraits et les plus complexes, ceux qui représentent des concepts ou des théories.

\section{Les images de base}

Lakoff a proposé une explication des débuts de la connaissance qui cadre bien avec la conception hiérarchique qu'on vient d'esquisser et permet de la préciser. Prenant appui sur la théorie des prototypes

1 M. DENIS et M. DE VEGA, "Modèles mentaux et imagerie mentale", in Les modèles mentaux. Approche cognitive des représentations, op. cit., p. 83.

2 P. LÉVY, L'idéographie dynamique, Genève, Le concept moderne/Édition, 1991, p. 87. 
élaborée par Rosch, Lakoff a élaboré une théorie de la connaissance basée sur l'expérience du monde telle qu'elle se construit à partir du corps et de la culture ${ }^{1}$.

Selon Lakoff, nos expériences corporelles préconceptuelles sont au fondement de toutes nos structures conceptuelles, même les plus abstraites. On trouve deux sortes de structures dans notre expérience préconceptuelle: les catégories du niveau de base (basic-level structures) et certaines images schématiques (kinesthetic imageschematic structures) et toutes deux procèdent de l'interaction du corps avec le monde.

La notion même de niveau de base est issue des travaux de Rosch et de ses disciples sur les catégories mentales. Elle signifie que parmi ces catégories, il en est qui sont primordiales, dans tous les sens du terme : elles surgissent en premier et constituent un fondement. Par exemple, des concepts comme "marteau", "chaise", "voiture", font partie du niveau de base mais non pas les catégories superordonnées "outil", "meuble", "véhicule", ou les catégories subordonnées désignant les différentes sortes possibles de marteaux, de chaises ou de voitures. Les concepts du niveau de base sont les premiers compris et nommés par les enfants ; ils correspondent à des gestalts perceptives se prêtant facilement à des interactions spécifiques et donnant lieu à de riches images mentales.

Les images schématiques, de leur côté, sont des structures simples, des schémas qui donnent sens à notre expérience quotidienne préalablement à toute autre imposition de concept : par exemple les schémas HAUT-BAS, DEVANT-DERRIERE, PARTIE-TOUT, CONTENANT-CONTENU, etc.

L'idée d'un niveau de base dont dérivent les autres niveaux permet en tout cas de donner une assise à l'idée de la connaissance comme élaboration d'images de différents niveaux de complexification et d'abstraction. Le niveau de base, c'est celui où les êtres parlent directement au corps, à sa capacité de s'identifier et de se mettre en correspondance avec eux. Une chaise est un objet proche que l'on peut éprouver comme ayant une physionomie -une forme à laquelle on peut s'identifier- modulée en fonction de certains usages possibles immédiatement ressentis.

1 Cf. G. LAKOFF, Women, Fire and Dangerous Things, What Categories reveal about the Mind, Chicago and London, The University of Chicago Press, 1987 ; et G. LAKOFF et M. JoHNSON, Les métaphores dans la vie quotidienne, Paris, Éd. de Minuit, 1985. 
Ce qui, toujours selon Lakoff, permet aux autres structures conceptuelles de s'élaborer à partir du niveau de base et des images schématiques, c'est essentiellement la métaphore comme processus de projection d'une structure conceptuelle sur une autre. On y viendra plus loin mais remarquons déjà qu'ainsi conçue, la métaphore constitue un aspect essentiel de la conceptualisation de l'expérience -donc de la raison humaine- et que le processus qu'elle désigne est intimement lié à l'image et à l'imagination.

What gives human beings the power of abstract reason ? Our answer is that human beings have what we will call a conceptualizing capacity. That capacity consists in :

- the ability to form symbolic structures that correlate with preconceptual structures in our every day experience. Such symbolic structures are basic-level and image-schematic concepts.

- the ability to project metaphoricaly from structures in the physical domain to structures in abstract domains (...) ${ }^{1}$.

\section{Le perspectivisme des images}

Il est une caractéristique des images sur laquelle on ne saurait trop insister : c'est leur perspectivisme, le fait qu'elles n'offrent jamais qu'un profil des choses qu'elles nous présentent. L'importance de ce fait se mesurera à l'explication qu'il nous offre de la très grande variété des connaissances que l'on peut prendre d'un même objet ou événement.

Le perspectivisme est un fait apparemment anodin car on peut le remarquer dans la perception la plus simple, laquelle suppose toujours un point de vue singulier d'où on regarde. "Les choses dans l'expérience naïve, écrivait Merleau-Ponty, sont évidentes comme êtres perspectifs (...) ; je saisis dans un aspect perspectif dont je sais qu'il n'est qu'un des aspects possibles, la chose même qui le transcende. Une transcendance pourtant ouverte à ma connaissance, c'est la définition même de la chose telle qu'elle est visée par la conscience naïve"2.

1 G. LAKOFF,Women..., op.cit., p. 281.

2 M. MeRleaU-PonTY, La structure du comportement, Paris, P.U.F., 1967, p. 202. 
La perception, donc, comporte une structure profil-tout -ou plus simplement partie-tout- et l'on peut penser que cette structure se répercute à travers toutes les images que l'on peut construire du réel.

Or une telle structure, notons-le, est paradoxale en ceci qu'elle signifie que la partie, dans une certaine mesure tout au moins, contient le tout. Elle peut du reste recevoir deux traductions antagonistes. D'une part, elle peut se dire : "face à une perception ou une image, on perçoit beaucoup plus qu'on ne sent ou ne voit effectivement". A l'opposé, elle peut s'énoncer comme ceci : "Une perception ou une image ne permettra jamais qu'une perception tronquée". La première traduction, au fond, renvoie à l'idée de signe ${ }^{1}$. La seconde traduction suppose quant à elle que toute connaissance est toujours partielle -et partiale- parce que toujours relative à un point de vue. La connaissance est toujours métonymique au sens où elle conceptualise une chose à travers ses parties. Et quel que soit le nombre de parties considérées, la chose elle-même restera toujours, comme dit MerleauPonty, une transcendance ouverte.

Mais cette proposition générale qui s'établit et se comprend facilement en référence au monde immédiatement perçu et aux images que l'on peut en construire, demande peut-être à être justifiée et précisée davantage pour les formes plus élaborées de représentation : les modèles mentaux qui, avons-nous vu, varient considérablement en complexité et en abstraction. C'est la notion de point de vue qu'il faut explorer ici.

On prendra toute la mesure de la très grande variabilité des points de vue et images correspondant à une même situation si l'on pense aux immenses possibilités de la langue dans la construction des images. Les mots, que l'on oppose systématiquement aux images, constituent en fait un instrument très raffiné de mise au point de l'imagerie par laquelle nous conceptualisons le monde.

Pour Langacker, avons-nous vu, les conventions lexicales et grammaticales utilisées pour représenter une situation opèrent la sélection de certains aspects, règlent leur saillance relative, la perspective sous laquelle ils sont vus, le niveau d'abstraction auquel on les considère, etc., etc. ${ }^{2}$. Les choses conceptualisées sont l'objet

1 Elle implique, soit dit en passant, que l'indice et l'icône ne sont ni séparables ni successifs mais sont plutôt contemporains ; mais on ne développera pas ici ce point.

2 Cf. LANGACKER, op. cit., chap. III. 
d'un ajustement focal que les expressions linguistiques ont à charge de préciser.

Toujours selon Langacker les mots se distribuent entre deux grandes catégories de prédicats définissables précisément en termes d'ajustement focal: les prédicats nominaux et les prédicats relationnels.

Les premiers, de manière prototypique, désignent des choses, et cela veut dire qu'ils mettent en profil des régions dans des domaines ou bases ("cercle" met en profil une configuration dans l'espace bidimentionnel). Par opposition, les prédicats relationnels mettent en profil des interconnexions et se partagent en deux groupes selon que celles-ci concernent un processus (ce qui est le cas des verbes) ou une relation intemporelle (ce qui est le cas des prépositions : au-dessus, avant, après...). Un verbe, par exemple, qui désigne un processus se déroulant dans le temps, implique des opérations cognitives de transformations successives d'une configuration en une autre.

$S$ 'il existe ainsi des noms, des verbes, des adjectifs, des prépositions, etc., etc., c'est, on le voit bien, pour rendre compte de toutes les mises en profil effectuées par la conceptualisation d'une chose ou d'un événement, d'une scène ou d'un ensemble de telles entités. C'est donc à tous les niveaux de la connaissance -conçue comme représentation iconique- que l'on trouve des mises en perspective, aussi bien au niveau des modèles mentaux abstraits qu'à celui des images mentales simples de l'environnement immédiat. D'une certaine manière, la connaissance se confond avec la mise en perspective. Produire une nouvelle connaissance, c'est réaliser une nouvelle mise en perspective mettant en saillance un aspect des choses jusque-là inaperçu ou considéré comme marginal, et reconsidérer l'ensemble des interconnexions à partir de ce point de vue inusité.

Un exemple. La deuxième topique freudienne a surgi d'une focalisation nouvelle et insistante sur certains aspects auparavant restés secondaires (le narcissisme, l'identification); et cette mise en perspective s'est résolue en une nouvelle image de l'architecture psychique centrée sur le moi mais comportant différentes zones en interaction : le ça comme zone active aux frontières floues, le moi comme région fermée aux contours plus nets, et le surmoi comme partie du moi reliée avec l'environnement social.

Dans cette perspective (c'est le cas de le dire), c'est la multiplication des points de vue qui est à la source des progrès de la connaissance. Dès son premier apprentissage de l'environnement, un sujet 
humain doit devenir capable de concevoir différents points de vue sur un même objet ; c'est d'ailleurs cela qui rend possible la notion même d'objet -comme transcendance- et permet au sujet le passage du je au $t u$ dans la relation interpersonnelle. Dans le domaine moins primitif de la connaissance scientifique, une transformation importante se caractérise souvent par la composition de plusieurs mises en profil. Dans l'exemple utilisé plus haut des topiques freudiennes, il semble évident que la deuxième de ces topiques intègre l'image de la première -issue d'une mise en profil de l'inconscient-dans une image remaniée.

Mais l'intégration des points de vue se fait toujours d'un point de vue et l'image -éventuellement très complexe- qui en résulte reste toujours une mise en profil. Une connaissance parfaitement objective, débarrassée de tout subjectivisme, c'est-à-dire élaborée hors de tout point de vue particulier, est impossible ${ }^{1}$.

La notion de point de vue est souvent vidée de ses implications spatiales et corporelles et réduite à la notion plus désincarnée d'opinion. Mais si la connaissance est image ou modèle mental, il faut insister sur ces implications. Même dans des domaines très abstraits, une représentation iconique suppose un ajustement focal -l'un ou l'autre profil saillant, une organisation figure/fond, etc.- issu d'un point de vue comme prise de position spatiale par rapport au domaine considéré et impliquant une attitude et un regard particuliers.

On peut supposer que le corps -dont on a déjà évoqué plus haut le rôle- est actif jusque dans les plus fins détails de la mise en profil d'un modèle mental. Les opérations cognitives correspondant aux prédicats nominaux ou relationnels ne peuvent se comprendre sans l'activité symbolisante du corps -contractions musculaires, impulsions d'actes...- et en particulier du regard. Dire qu'un prédicat nominal met en profil une région dans un domaine suppose un regard pour l'isoler, en fixer les limites, lui donner corps par identification au corps propre-au dedans qu'il représente par opposition à un dehors ${ }^{2}$. Dire qu'un verbe, comme prédicat relationnel séquentiel, met en profil un processus suppose une activité motrice symbolisant ce

1 Pour Langacker comme pour Lakoff ou Putnam, l'objectivité est synonyme de multiplication des points de vue. Cf. à ce sujet G. LAKOFF, Women..., op. cit., Part II.

2 Pour Lakoff et Johnson, le corps implique un schème contenant/contenu ou dedans/dehors qui se trouve à l'origine de métaphores ontologiques donnant corps à des entités qui, sans cette projection, n'en auraient pas. 
processus. La signification du verbe marcher, remarque Langacker, doit comprendre une image motrice correspondant à la marche.

Ainsi la construction d'un modèle mental à travers un discours comportant des noms, des verbes, des adjectifs, des prépositions, etc. témoigne d'une multiplicité de mises en profil de régions, de relations statiques ou de transformations dynamiques, plus ou moins saillantes. Une perspective globale en émerge, point de départ et fin d'un processus de construction impliquant le corps et son activité de symbolisation élective à la fois globale et différenciée.

Donc, à quelque niveau d'abstraction qu'on l'envisage, la connaissance procède par mises en perspective et ajustement focal. Les profils des états de chose qui en résultent sont en principe infinis et peuvent -comme on l'a vu sur l'exemple de la seconde topique freudienne- s'intégrer ou s'enchâsser les uns dans les autres.

On pourra objecter à cela que dans une pareille épistémologie, tout est toujours bon ou que rien n'est véritablement réfutable car une mise en profil n'en exclut aucune autre et ne peut être réfutée par aucune autre. C'est effectivement une idée que rend très plausible la conception perspectiviste de la connaissance. La connaissance ne semble pas devoir nécessairement progresser par un jeu d'argumentation, réfutation, coupure... ; si elle est mise en profil, elle ne peut qu'avancer par intégration de profils successifs. S'il lui est nécessaire de remettre quelquefois en cause les perspectives dominantes -par un jeu de réfutation et d'affirmation-, elle doit néanmoins faire leur part aux aspects des choses rendus saillants par ceux-ci, quitte à revoir leur place dans un nouveau modèle. S'il n'y a pas de point de vue définitif mais une infinité de points de vue et de profils possibles, la connaissance ne peut progresser que par complexification et non par négation- affirmation.

\section{Assimilation, comparaison, métaphorisation}

On sait que pour Piaget l'assimilation est au fondement de l'activité cognitive. Qu'il s'agisse de l'intelligence sensori-motrice ou du jugement, l'adaptation intellectuelle consiste à faire rentrer le nouveau dans le connu. Toute perception nouvelle est intégrée à une structure antérieure ; elle dépend donc autant de l'expérience ancienne que du stimulus (ou input). 
Langacker dit quelque chose de très semblable lorsqu'il fait de la comparaison un concept fondamental pour caractériser la vie mentale :

Fondamental to cognitive processing is our ability to compar events and register any contrast or discrepancy between them ${ }^{1}$.

Pour lui comme pour Piaget, une expérience mentale ne dépend pas seulement de l'input, elle dépend aussi des structures que lui impose nos attentes et routines interprétatives.

On voit clairement que des concepts qui, comme ceux de comparaison ou d'assimilation, impliquent la compréhension d'une chose (disons, comme Langacker, d'une cible T) au moyen d'une autre chose (d'une source ou entité servant de standard S), sont indispensables au constructivisme ${ }^{2}$. En particulier, ils sont indispensables à une perspective faisant de l'imagerie et de la mise en profil l'essentiel de la connaissance. Rendre saillants tels aspects d'une entité (chose, événement, processus...) suppose que soient effectuées un grand nombre de comparaisons à l'intérieur de l'entité (entre les différents aspects) et entre cette entité et d'autres. L'importance de tels concepts vient de ce qu'on peut en dériver certains modes de conceptualisation caractéristiques de la vie mentale : la métaphorisation, la schématisation et certaines opérations inférentielles qui en découlent.

On a déjà vu plus haut que pour Lakoff, la base de la connaissance est constituée par les deux sortes de structures préconceptuelles : les catégories du niveau de base (basic-level structure) et les images schématiques (kinesthetic image-schematic structure). Les autres catégories -notamment les plus abstraites- émergent par projection métaphorique. C'est une corrélation entre deux expériences qui permet cette projection de l'une (que l'on appelera la source) sur l'autre (la cible); et une corrélation, perçue ou vécue, repose nécessairement sur une comparaison.

"L'essence d'une métaphore, écrivent Lakoff et Johnson, est qu'elle permet de comprendre quelque chose (et d'en faire l'expérience) en termes de quelque chose d'autre"3. Il ne s'agit pas ici de

1 Op. cit., p. 101.

2 La notion d'assimilation est peut-être plus fondamentale dans la mesure où elle réfère à une sorte d'indifférenciation primitive alors que la notion de comparaison suppose une séparation des termes comparés. Mais on ne tiendra pas compte ici de ces nuances.

3 La métaphore..., op. cit., p. 15. 
simples jeux de mots ou figures de style mais d'un processus fondamental de conceptualisation dont le langage ordinaire porte les traces. Lakoff et Johnson distinguent plusieurs formes de ces métaphores conceptuelles : les métaphores d'orientation, les métaphores ontologiques et les métaphores structurales. Un exemple des premières est donné par des expressions telles que : "Je suis aux anges" ou "ça m'a remonté le moral". Elles impliquent la métaphore conceptuelle sousjacente : le bonheur est en haut qui donne structure à une émotion à partir de l'image schématique haut-bas relative à notre expérience corporelle du monde. Les métaphores ontologiques nous permettent de percevoir des événements, des émotions ou des idées comme des substances ; c'est par elles que l'on peut concevoir comme des objets possédant des formes distinctes des choses qui n'ont que des limites floues : des forêts, des clairières, des professions, des domaines de connaissance... Les métaphores structurales utilisent un concept hautement structuré pour en structurer un autre. Par exemple, le langage quotidien comporte un grand nombre d'expressions montrant de toute évidence une assimilation du concept de discussion au concept de guerre :

Vos affirmations sont indéfendables. Il a attaqué chaque point faible de mon argumentation. Ses critiques visaient droit au but. J'ai démoli son argumentation $(\ldots)^{1}$.

Le concept de guerre, comme "espèce naturelle d'expérience" est une "gestalt expérientielle" possédant une structure interne comportant différentes composantes : défi, intimidation, conquête, défense, retraite, reddition, etc. C'est cette gestalt qui est projetée sur celle de conversation lorsque celle-ci, gagnant en animation, tourne à la discussion.

Comprendre, c'est donc appliquer une gestalt sur une autre ; et le résultat de cette application, c'est, dans la terminologie que nous avons empruntée à Langacker -une mise en profil. Lakoff et Johnson soulignent du reste le caractère partiel d'une structuration métaphorique, le fait qu'elle met en relief certains aspects d'une gestalt et en masque d'autres (les aspects coopératifs de la discussion).

Ce qui se passe, en fait, dans la projection de la structure-source sur la structure-cible est en réalité très complexe, comme en témoignent ces belles lignes dans lesquelles Lakoff et Johnson tentent de

1 Ibid., p. 14. 
décrire ce qui se passerait dans notre conception de l'amour si l'on substituait aux métaphores habituelles une nouvelle, qui en appellerait à l'art :

La métaphore L'AMOUR EST UNE CEUVRE D'ART RÉALISEEE EN COMMUN offre un exemple subtil de similitudes créées par une métaphore nouvelle. Elle met en valeur certains aspects des expériences amoureuses, atténue le rôle d'autres aspects et en masque d'autres encore. En particulier, elle atténue le rôle des expériences qui correspondent à la métaphore L'AMOUR EST UNE FORCE PHYSIQUE. Par "atténuer le rôle", nous voulons dire qu'elle est logiquement compatible avec certaines expériences de l'amour qui pourraient très bien être exprimées par "Il existe entre nous un flux magnétique", "le courant passe", etc. ; elle n'attire cependant pas l'attention sur celles-ci. D'un autre côté, elle masque les expériences de l'amour qui correspondent à la métaphore L'AMOUR C'EST LA GUERRE, car il n'y a pas de chevauchement possible entre les deux métaphores. Les aspects de collaboration et de coopération de la métaphore L'AMOUR EST UNE CEUVRE D'ART RÉALISÉE EN COMMUN sont incompatibles avec les aspects agressifs et dominateurs de nos expériences amoureuses qui peuvent être exprimés par : "c'est ma dernière conquête", "il s'est livré à elle", "elle m'a accablé de ses assauts", etc. Par ce moyen (...) la métaphore met entre parenthèses certaines de nos expériences amoureuses et en désigne d'autres à l'attention comme si elles étaient seules pertinentes. Ce faisant, elle induit un ensemble de similitudes entre nos expériences amoureuses et les expériences réelles et imaginaires de collaboration à une œuvre d' $\operatorname{art}^{1}$.

On ne peut mieux caractériser la mise en profil en quoi consiste la projection métaphorique.

La métaphore induit une modification globale de points de vue qui rejaillit sur tous les aspects du modèle mental; elle est une comparaison ou superposition créant des résonances qui remodèlent toute la structure figure/fond de l'image-cible - ce qui en outre ne peut manquer d'avoir quelque effet en retour sur l'image-source. Si, comme dans la célèbre métaphore cinématographique de Modern Times, de Chaplin, on fait se succéder l'image d'un troupeau de moutons et l'image d'une foule humaine, celle-ci sera saisie à travers la première et il s'ensuivra une mise en profil focalisant sur le gréga-

1 Ibid., p. 159. 
risme des individus de la foule, sur leur comportement qui consiste à se laisser conduire et dominer par le mouvement du groupe. Autrement dit : la reprise mimétique du comportement moutonnier sera projetée sur le comportement humain, mettant à l'avant-plan ce que ce dernier peut avoir de grégaire, et repoussant du même coup vers l'arrière-plan les autres composantes de ce même comportement. On voit bien, sur ce dernier exemple, le lien entre la métaphore, le caractère mimétique de l'image dont il a été question plus haut, et la mise en profil. C'est la transposition, d'une image à l'autre, d'une activité mimétique similaire -comportant : mouvements symboliques du corps et de ses parties, ébauches motrices, projection, identification...-qui induit la similitude de mise en profil. La transposition du comportement du mouton sur celui de l'individu humain se fait par l'intermédiaire de la capacité mimétique qui structure l'image. Il en est de même pour le transfert du comportement de l'artiste sur celui de l'amoureux, transfert qui met en saillance dans l'image de ce dernier celles de ses conduites qui se coulent dans le modèle identificatoire de l'artiste.

La métaphore crée-t-elle ou révèle-t-elle les similitudes ? La métaphore est difficilement concevable sans un minimum de similitude, quelques traits auxquels peut accrocher l'activité fondamentale de comparaison. Mais elle est aussi créatrice dans la mesure où, sous son action, ces traits communs vont passer à l'avant-plan, gagner du relief et induire une recomposition de l'ensemble des traits. La révélation est en quelque sorte une création.

La projection métaphorique n'est cependant qu'une des issues de la comparaison. Elle est du reste logiquement liée à d'autres issues possibles : l'identification ou son contraire, la différenciation, la schématisation. Ce dernier concept, on va le voir, mérite une attention toute particulière.

\section{Schéma et schématisation}

Ces notions ont déjà une certaine histoire. Elles ont trouvé place dans de nombreux textes consacrés à l'activité cognitive, en psychologie comme en philosophie ${ }^{1}$. Par exemple, on peut trouver chez Kant

${ }^{1}$ Cf. R. Estivals, «Théorie cognitive de la schématisation», Schéma et schématisation, revue de schématologie et de bibliologie, $\mathrm{n}^{\circ} 46$. On trouvera dans 
une théorie du schème et de la schématisation destinée à rendre compte du passage du sensible au concept. On sait par ailleurs l'importance attribuée par le psychologue Piaget à la notion de schème et en particulier de schème sensori-moteur, sorte de canevas de rapport à l'objet qui conduira au pré-concept puis au concept. Chez ces auteurs, le schème apparaît comme une sorte d'intermédiaire entre le particulier et le général ou entre le concret et l'abstrait. Cette même caractéristique d'entre-deux se retrouve, on va le voir, dans la notion de schéma apparue récemment dans le cadre de la psychologie cognitive. L'idée de schéma a été introduite -par des auteurs comme Rumelhart, Ortony, Norman- pour désigner des structures de représentation plus complexes que celles correspondant au mot et à la phrase.

Ces unités plus larges sont décrites ainsi par Rumelhart et Norman :

Les schémas sont des structures de données destinées à représenter les concepts génériques stockés en mémoire. Il existe des schémas pour les concepts généraux qui sont sous-jacents aux objets, aux situations, aux événements, aux actions et aux séquences d'actions : un ensemble de relations dont on pense qu'il fait partie des constituants des concepts qu'il représente. Les schémas, en quelque sorte, représentent les stéréotypes de ces concepts. Pour simplifier, ils sont comme des modèles du monde extérieur. Traiter une information à l'aide d'un schéma revient à déterminer quel est le modèle qui convient le mieux à l'information d'entrée (input) $^{1}$.

Les schémas sont des "paquets d'information" dépassant largement ce que l'on peut trouver dans les dictionnaires au sujet du sens des mots. Des concepts comme "oiseau" ou "chien" comportent tout un ensemble de faits et de relations en rapport avec ces animaux. Les schémas, disent Rumelhart et Norman, sont nos connaissances: "Toutes nos connaissances génériques sont enchâssées dans des schémas ${ }^{2}$.

cet article une intéressante mise en perspective de l'histoire du concept chez des auteurs comme Kant, Bergson, Sartre, Piaget, etc.

1 D. Rumelhart et D. NormaN, "Representing Memory" repris dans $L a$ psychologie, op. cit., p. 310.

2 Ibid. p. 312. 
Les schémas ont un aspect stéréotype, comme l'indique clairement la définition de Rumelhart et Norman, mais l'aspect qui nous intéresse le plus, ici, et dans lequel, d'ailleurs, on va retrouver cette caractéristique d'entre-deux évoquée plus haut, réside dans le fait qu'ils contiennent une partie constante et une partie variable. Au mot "donner" par exemple, correspond un schéma comportant une partie constante représentant un changement de possesseur et comme partie variable les caractéristiques de celui qui donne et de celui qui reçoit. Ce rapport entre le constant et le variable indique une propriété fondamentale des modèles mentaux qui est de varier en spécificité.

Dans cette perspective, Langacker donne de la schématicité une définition fondée sur la disparition progressive des détails :

The notion of schematicity pertains to level of specificity, i.e. the fineness of details with which something is characterized; the notion allways pertains, primarily if not solely, to precision of specification along one or more parameters, hence to the degree of restriction imposed on possible values along these parameters ${ }^{1}$.

La schématicité est la manifestation d'une aptitude fondamentale à la sélection et à l'abstraction; mais cette dernière ne se comprend pas si on ne la relie à celles d'assimilation et de comparaison déjà envisagées plus haut. Toutes ces notions sont interdépendantes et expliquent -comme on le verra un peu plus loin-, certaines formes fondamentales de raisonnement fondées sur l'iconicité.

Dans la grammaire cognitive de Langacker, ce sont les comparaisons entre images-concepts qui impliquent la formation de concepts plus schématiques et au-delà, de réseaux schématiques de concepts interreliés. Il vaut la peine de résumer ici ces processus car, au-delà de la formation des catégories lexicales ou grammaticales -objet principal de la recherche de Langacker- ils concernent la formation et l'organisation des connaissances en général.

Soit l'apprentissage, chez un enfant, de la catégorie lexicale ARBRE avec ses différents sens interreliés. Le point de départ réside dans la constitution d'un prototype (PT), c'est-à-dire un schéma de bas niveau correspondant aux aspects communs de spécimens ordinaires tels les chênes et les ormes (correspondant au niveau de base de E. Rosch). Lors de la rencontre avec un arbre aussi différent du proto-

1 Ibid, p. 132. 
type que l'est le pin, il se produit une extension axée sur un jugement de catégorisation (arbre $\longrightarrow$ pin) fondée sur la perception d'une similarité induisant un concept (arbre') intégrant la similitude entre arbre et pin. L'expérience se poursuivant par la rencontre d'autres arbres (comme le palmier) posant de nouveaux problèmes d'intégration, il s'ensuivra une complexification de la catégorie lexicale sous forme d'un réseau dont l'expansion dans le sens horizontal correspond à l'extension à partir d'un prototype et dont l'expansion dans le sens vertical correspond à l'extraction de schémas successifs. C'est ce que montre le schéma suivant, repris à Langacker ${ }^{1}$ :

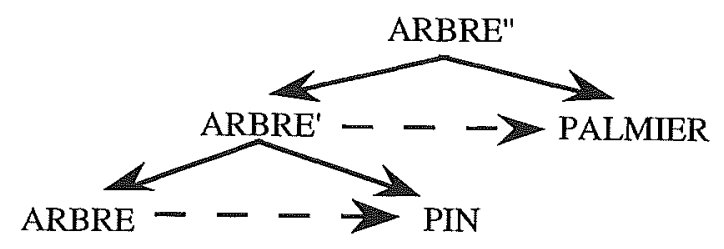

Des réseaux lexicaux de cette sorte peuvent devenir extrêmement complexes : comporter des nœuds et relations plus saillants que d'autres, comporter des prototypes ou des schémas locaux, dans des sous-configurations, etc. Ils sont aussi dynamiques et changeants parce que soumis aux pressions constantes de l'expérience et de la communication.

Mais ce qui importe ici, avant tout, c'est l'interdépendance entre les opérations cognitives figurées par le triangle de base :

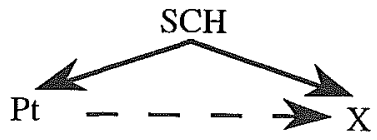

La perception d'une similarité entre un prototype et une autre entité $(\mathrm{Pt} \longrightarrow \mathrm{X})$ suppose une troisième entité cognitive : le schéma $\mathrm{SCH}$ que $\mathrm{Pt}$ et $\mathrm{X}$ élaborent de manière alternative. L'abstraction qui sous-tend l'extraction du schéma suppose qu'à travers la comparaison des deux entités, certaines spécifications de $\mathrm{Pt}$ soient suspendues ou relâchées tandis que d'autres deviennent plus saillantes. Ce mécanisme, on le voit, recoupe celui mis au jour par

1 op. cit., p. 374. 
Lakoff au sujet de la métaphore comme projection d'un domainesource sur un domaine-cible mettant en saillance certains éléments et en effaçant d'autres. Cette projection implique effectivement la mise en profil d'une partie perçue comme similaire : le schéma avec sa partie constante (commune aux différentes entités comparées) et sa partie variable (les éléments susceptibles de spécification différentes dans les diverses entités concernées). La schématisation est en quelque sorte un produit de la comparaison comme le sont, du reste, l'extension (assimilation à une même catégorie) et la métaphorisation (superposition partielle de catégories restant différenciées. Le schéma qui en découle, notons-le, n'acquiert pas forcément une existence autonome.

La schématisation est aussi corrélative d'une certaine activité inférentielle. Telle que décrite par Lakoff, la métaphore, on l'a vu plus haut, implique déjà une certaine forme d'inférence consistant en la recherche de toutes les implications procédant de l'interférence entre deux gestalts : la conception de l'amour comme d'une ouvre d'art provoque des résonances entre deux tableaux entraînant certaines implications (qui seront ou non exprimées): par exemple, que l'amour est une espèce de travail, mais pas un travail quelconque : "Il s'agit d'un travail qui exige cet équilibre particulier entre contrôle et passivité qui convient à la création artistique, puisque le but poursuivi n'est pas n'importe lequel mais un but artistique commun"1.

Mais la schématisation autorise une démarche un peu plus compliquée bien que fondamentalement de même nature. Voici un exemple qui présente, outre l'avantage de concerner directement à la fois les images mentales et les les images matérielles, celui de montrer les aléas et dérapages possibles de l'opération inférentielle. Dans son étude sur l'imagerie initiale, B. Darras appelle "iconotype" le type d'image prévalant dans la représentation picturale enfantine. L'iconotype est à mettre en rapport avec la formation des catégories cognitives et le niveau de base tel que caractérisé par E. Rosch (cf. plus haut). Adressée à un enfant, la consigne "dessine une fleur" déclenche généralement la production d'une fleur typique comprenant les caractéristiques saillantes de la catégorie : un centre, une tige (généralement en un seul trait), des pétales (le plus souvent cinq) et deux feuilles symétriques. Il s'agit là d'une transposition dans le domaine graphique des attributs de la catégorie cognitive. Mais le

1 G. LAKOFF, Les métaphores ..., op. cit., p. 151. 
processus aboutit quelquefois à des résultats étranges. Lors d'une expérimentation portant sur de nombreux enfants (2 900 de différents pays) et ayant pour consigne "Dessine un coq qui marche devant un arbre", plusieurs d'entre eux dessinent plus de deux pattes à leur coq et en général quatre. L'explication que donne B. Darras est pleine d'intérêt pour le processus inférentiel qui nous intéresse pour le moment. Il est probable, remarque-t-il d'abord, que le coq, cet animal peu connu des petits urbains, occupe en plus une position marginale dans la catégorie oiseau et même que pour certains, il n'en fasse pas partie. D'où la méprise possible : "les dessinateurs qui ne disposent pas des informations du niveau de base doivent les collecter à un niveau plus général encore. Cette situation a dû concerner beaucoup d'enfants. Pour eux, la tâche réclamait la constitution d'un schéma à partir d'une catégorie de niveau surordonné. Ceux qui ont choisi d'accéder au niveau surordonné «oiseau» ont produit un dessin relativement conforme aux attentes des expérimentateurs. En conséquence, leur dessin est celui d'un oiseau type auquel ont été éventuellement greffés quelques attributs du coq, c'est-à-dire généralement une crête. Pour ceux, dont le niveau surordonné est celui de l'animal générique et son anatomie standard composée de quatre pattes, le résultat graphique est celui qui nous a tant surpris"1.

L'exemple est riche d'indications sur un processus dont l'essentiel peut peut-être se caractériser comme : activation d'un ou de plusieurs schéma(s) sur base d'une analogie perçue ou imaginée et remplissage par des caractéristiques associées par contiguité2.

\section{Aspects de la connaissance ordinaire}

Les considérations précédentes conduisent à concevoir la connaissance comme un vaste réseau d'images ou modèles reliés entre eux par des liens associatifs fondés sur la similitude (métaphore conceptuelle au sens de Lakoff) ainsi que sur la contiguité. Une hiérarchie de niveaux de schématicité structure également ce réseau

1 B. DARRAS, Au commencement était l'image, du dessin d'enfant à la communication de l'adulte, Paris, ESF éditeur, 1996, pp. 143-144.

2 Dans la théorie des schémas, soutenue par le modèle connexionniste de la cognition, les connaissances représentées n'apparaissent pas selon la règle du tout ou rien; plusieurs schémas peuvent être activés et c'est celui qui; dans la dynamique du système, comportant activations réciproques et inhibitions, restera le plus activé qui restera activé. 
qui se déploie, rappelons-le, autour d'un niveau de base dont la constitution s'enracine dans l'expérience corporelle du monde. Si donc la schématicité est une notion relative -on peut toujours spécifier ou schématiser davantage un modèle-, on notera cependant qu'elle gagne en degré au fur et à mesure qu'on s'éloigne du niveau de base vers le haut. Le schéma triangulaire de Langacker $(\mathrm{Pt}-\mathrm{SCH}-\mathrm{X})$ constitue un bon résumé synthétique de cette structuration en réseau issue tout entière d'une activité cognitive fondamentale de comparaison ; on y substituera cependant, à la notion de prototype $(\mathrm{Pt})$ qui réfère au modèle central des catégories lexicales, le terme plus général de modèle, lequel renvoie à toute forme de connaissance, qu'elle soit ou non encodée dans le lexique et quel que soit son niveau d'abstraction. On obtient ainsi :

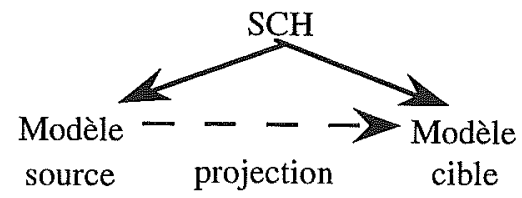

On ne prétendra pas que la cognition se réduit tout entière à cette structure, à sa construction et à son type de fonctionnement inférentiel. On dira simplement que cette structure semble bien correspondre à quelque aspect essentiel de ce que nous livre une description phénoménologique de notre expérience mentale de sujet connaissant. Or, du point de vue des sciences de la communication, cette expérience mentale accessible à la conscience est évidemment essentielle. Il importe plus de savoir quelle expérience de conscience accompagne l'activité de l'homme communiquant que de reconstruire les processus cognitifs inconscients supposés sous-jacents ${ }^{1}$. Et ceci non seulement au nom de la réalité de l'expérience mentale mais aussi parce qu'elle est la seule réalité qui a une importance dans la vie pratique, individuelle ou sociale.

On peut illustrer cette dernière idée par une description de certaines des formes de notre connaissance, telles que nous les livre notre expérience de sujet connaissant, et telles que nous permet de les reconstruire la structure proposée.

1 Concernant cette problématique, on trouvera beaucoup d'éléments de discussion dans F. VARELA, E. Thomson, E. RosCh, L'inscription corporelle de l'esprit, Paris, Éd. du Seuil, 1993. 
Nous disposons, pour régler nos rapports au monde et communiquer de quantités de modèles plus ou moins schématiques qui peuvent s'assembler et se spécifier selon les situations, les problèmes à résoudre, les raisonnements à effectuer... Il s'en faut cependant que ces modèles soient homogènes. Il est pour chacun de nous des zones de connaissances pour lesquelles nous disposons de nombreux modèles fortement interconnectés et hiérarchisés. Il en est d'autres qui ne comportent que quelques modèles très schématiques et faiblement interconnectés. Les savoirs qui concernent les objets et situations de la vie quotidienne sont certainement, pour chacun de nous, les mieux structurés. Les schémas et sous-schémas que comportent ces savoirs se sont construits par schématisations successives à partir des "gestalts" prégnantes et fréquemment perçues du niveau de base (cf. plus haut). Ils permettent des inférences relativement sûres et sont suffisamment souples pour se spécifier de multiples façons. Ce sont ces schémas qui fournissent leurs exemples privilégiés aux théoriciens de la cognition. Le schéma correspondant au verbe "donner" par exemple comporte au minimum l'image d'un mouvement de desaisissement se produisant entre deux entités très abstraites et c'est cette image qui nous guide dans le repérage des situations auxquelles on peut appliquer le verbe. Mais cette image peut se spécifier de multiples façons en fonction des choses et des personnes concrétisant l'échange ou en fonction d'aspects psychologiques, sociaux, juridiques, affectifs, etc., qui font qu'un don peut être un bienfait, une faveur, une attribution, etc., et peut comporter diverses sortes d'obligations et de conséquences : gratitude, amitié, subordination, ressentiment... L'image du désinvestissement comporte toutes ces spécifications possibles comme autant de sous-schémas prêts à se développer selon les circonstances, le contexte, les indications que donnent les situations concrètes... La richesse d'un schéma est fonction de la diversité des sous-schémas qu'il contient potentiellement et qui orientent nos hypothèses.

Il est vraisemblable que les zones du savoirs qui ne relèvent pas directement de la vie quotidienne mais qui ont fait l'objet d'un apprentissage systématique soient structurés de la même manière. Les scientifiques disposent sans doute au sujet de leur domaine d'études d'une hiérarchie complexe de modèles schématiques construits à partir des éléments de base du domaine. Mais que se passe-t-il dans les zones du savoir très éloignées de la vie quotidienne et qui n'ont pas fait l'objet d'un apprentissage systématique? Ces zones se sont 
considérablement développées grâce aux médias, lesquels diffusent quantités d'informations sur toutes sortes de domaines souvent très éloignés de la vie ordinaire (l'astrophysique, la biologie cellulaire, la climatologie, l'ethnologie, etc.) mais pouvant néanmoins avoir un impact considérable sur celle-ci (l'économie par exemple).

On peut faire l'hypothèse que les connaissances concernant ces domaines relèvent de schémas dont la portée inférentielle reste très limitée. Ils ne reposent pas, généralement, sur des niveaux plus basiques dont ils auraient été extraits et en conséquence, ils sont pauvres en sous-schémas pouvant se spécifier de diverses façons selon les circonstances. Pour un spectateur de télévision moyen, la notion d'anticyclone est associée à celle de beau temps selon une relation vague dans laquelle intervient la pression atmosphérique, mais faute de pouvoir aller très loin dans les questions relatives à la pression atmosphérique, et à ses rapports avec le temps, il ne pourra guère spécifier et nuancer ses prédictions.

Une autre caractéristique de tels schémas est, souvent, de ne mettre en profil que certains aspects partiels et quelquefois marginaux des phénomènes. Pour un philosophe ou un sociologue, la notion de "prolétariat" prend place immédiatement dans un modèle mental comportant au moins : une autre place pour les capitalistes et une mise en scène de leurs rapports ; il sera en outre capable de spécifier ce schéma en suivant les voies offertes par la théorie ou par l'histoire des faits réels ; Or, voici la représentation que donne un sujet interrogé par un psychologue ${ }^{1}$ au sujet de termes usuels :

Prolétariat: j'avais une image étrange, une étendue plate et noire, et, au-dessous, une mer roulant obscurément, un flot indéterminé, quelque chose comme une masse sombre et épaisse roulant de lourdes vagues. Que signifiait la masse? L'extension dans le monde entier; quelque chose comme un dynamisme latent.

Sartre tire beaucoup d'enseignements de ce type de schéma symbolique au sujet de l'acte de compréhension intuitive. Mais du point de vue adopté ici, l'exemple est également riche d'indications. Il met en évidence le jeu des projections métaphoriques à un niveau très élémentaire : le prolétariat et les vagues sont assimilés l'un à l'autre à travers le mouvement corporel qui les mime l'un et l'autre. Il montre

1 Flash, cité par J.-P. SARTRE dans L'imaginaire, Paris, Gallimard, 1940, p. 193. 
aussi la centration excessive sur un aspect des choses qu'une mise en perspective plus distante aurait relativisé.

Un dernier exemple pour illustrer la marginalité de certains schémas. Pour un économiste ou pour quelqu'un ayant quelques connaissances en économie, l'inflation est une notion relativement complexe induisant un modèle schématique comportant un phénomène central (la hausse des prix) et autour de ce centre de l'attention, toutes sortes d'entités et de relations schématiques : les coûts de production, les charges salariales, la demande, les capacités de la production, le volume des moyens de paiement, etc. Selon les besoins et les circonstances, chacune de ces entités ou relations peut être mise en profil et donner lieu à des spécifications plus ou moins détaillées.

Mais pour l'individu qui ne possède que les connaissances de l'homme de la rue -connaissances souvent simplement relayées par la grande presse-, le modèle de l'inflation se réduit à peu de chose. Lakoff et Johnson ont relevé de nombreuses expressions linguistiques montrant la réduction métaphorique de l'inflation à une chose contre laquelle il faut lutter :

Prenons l'expérience de la hausse des prix, qui peut être métaphoriquement considérée comme une entité sous le nom d'inflation. L'INFLATION EST UNE ENTITÉ permet de nous référer à l'expérience ;

L'inflation est en train de faire baisser notre niveau de vie. S'il y a encore plus d'inflation, nous ne survivrons jamais. Il faut combattre l'inflation (...)

Dans tous les cas, considérer l'inflation comme une entité nous permet d'y faire référence, de la quantifier, d'en identifier un aspect particulier, de voir en elle une cause, d'agir en tenant compte d'elle et peut être même de croire que nous la comprenons ${ }^{1}$.

La "chosification" de l'inflation ne réussit en fait à mettre en évidence, au-delà de son existence comme phénomène localisable (sinon dans les choses elles-mêmes, au moins dans un modèle mental de ces choses), que son impact négatif sur le niveau de vie, lequel est un aspect périphérique qui ne permet d'entrevoir pratiquement rien de l'ensemble des phénomènes économiques liés à la hausse des prix ${ }^{2}$.

1 G. LAKOFF et M. JOHNSON, op. cit., p. 36.

2 Ce dernier exemple laisse entrevoir l'intérêt des notions évoquées dans le cadre de la communication des savoirs. De ce point de vue, le triangle modèle-source modèle-cible — schéma constitue un modèle permettant de mettre en profil 


\section{Conclusions : vers une sémiotique cognitive}

L'idée -schématique- d'une iconicité de la pensée nous a fait effectuer un parcours que l'on peut résumer brièvement en quelques points s'impliquant mutuellement.

1. La connaissance commence avec la duplication mimétique du réel perçu. Elle se développe ensuite à travers une hiérarchie de représentations iconiques allant des images du niveau de base (images des entités à portée du corps) vers des "modèles mentaux" de plus en plus abstraits mais néanmoins toujours iconiques (et compris comme tels à travers les capacités mimétiques du corps).

2. La connaissance par icônes est corrélativement une connaissance par profils, ce qui veut dire que toute image ou modèle rend saillants certains aspects des phénomènes et en efface d'autres.

3. Dans la mesure où la connaissance repose sur une activité fondamentale d'assimilation ou de comparaison, elle procède nécessairement par métaphorisation et schématisation. La notion de schéma (cas particulier de la mise en profil) est apparue capitale et conduit à une conception de la connaissance comme un vaste réseau de modèles schématiques plus ou moins enchâssés les uns dans les autres et donc plus ou moins spécifiables, ces deux "plus ou moins" rendant compte des variations du savoir selon son degré de systématicité.

Il nous faut maintenant envisager les voies qu'ouvrent ces quelques idées à une sémiotique cherchant à se développer du côté de la cognition.

Pris à son niveau le plus fondamental, le problème est de préciser l'articulation entre les signes externes (essentiellement le langage verbal et les images matérielles) et les images et modèles mentaux que l'on peut considérer comme des représentations ou signes internes.

Cette articulation, dans la perspective adoptée ici, ne peut être conçue comme simple traduction. La pensée et ses signes ne se développent pas d'abord pour se sémiotiser ensuite. Les signes externes,

certaines des conditions sous lesquelles un schéma peut servir à la compréhension et à la maîtrise du réel. On peut en déduire une règle générale que l'on peut formuler ainsi : la projection d'un modèle-source sur un modèle-cible doit autant que possible aboutir à la formation d'une image schématique du second mettant en profil quelques-uns de ses aspects centraux et permettant d'inférer un maximum de spécifications potentielles. 
autrement dit, ne sont pas de simples moyens de communication des signes internes. Ils sont aussi, selon la belle expression de J. Goody, des "technologies de l'intelligence", des outils externes dont l'usage infléchit les formes et les opérations de la pensée ${ }^{1}$. Cette idée est portée par tout un courant de pensée qui place le fonctionnement cognitif sous la dépendance de la médiation sémiotique, ou plus précisément sociosémiotique puisque les signes sont un produit des interactions sociales ${ }^{2}$. Dans le cadre de ce courant théorique, l'hypothèse à explorer est celle d'une relation circulaire faisant dépendre l'un de l'autre le sociosémiotique et le psychologique. Mais on sait que cette circularité pose quelques difficiles problèmes de méthode. Pour penser la circularité, il faut dissocier quelque peu les termes (ici, la pensée et les signes) pour reprendre ensuite le problème de leur détermination réciproque. C'est ce qu'on a fait ici en envisageant tout d'abord la pensée, choix motivé par l'idée que, relativement aux langages, la pensée paraît bien primordiale et liée à notre rapport originairement corporel (sensori-moteur) au monde. C'est par ailleurs le rôle fondamental de la médiation corporelle dans la formation de la pensée qui donne les meilleurs arguments en faveur de l'intuition du caractère primordialement iconique de la pensée. Reste donc maintenant à examiner le second terme et ses effets en retour sur nos pensées-images. Reste, autrement dit, à aborder le problème de la codétermination de celles-ci par la médiation sémiotique ${ }^{3}$. Concernant ce sujet très vaste, on se limitera ici au repérage de certaines des questions constitutives de la problématique. Un schéma synthétique peut aider à ce repérage :

1 Cf. J. Goody, La raison graphique, Paris, Éd. de Minuit, 1979.

2 Se rattachent à ce courant, outre l'anthropologue J. Goody, des auteurs comme le psychologue du développement Vygotsky ou le philosophe P. Lévy qui lui a donné tout récemment une expression particulièrement forte et intéressante. On peut également lui associer la perspective médiologique.

3 Les problèmes liés à la notion de circularité mériteraient sans aucun doute un examen beaucoup plus approfondi. 


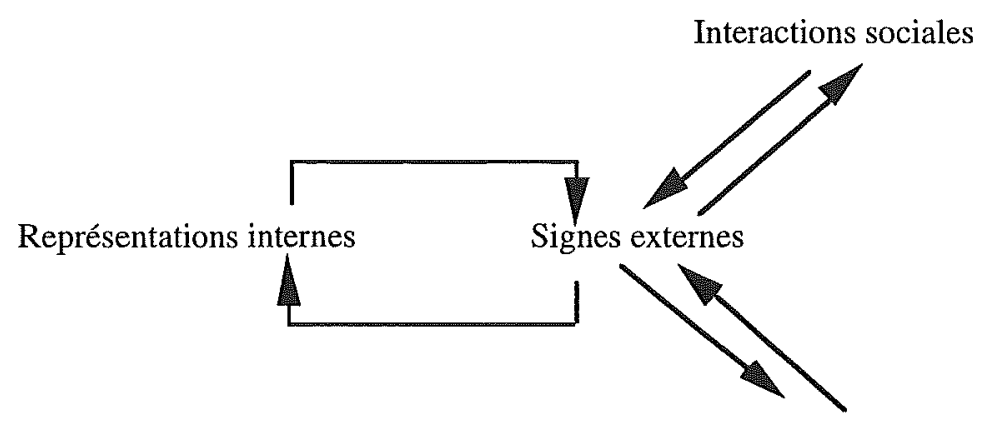

Techniques

On a déjà eu l'occasion d'envisager dans cette perspective le langage verbal. Une fois reconnue les fins iconiques du langage verbal, avons-nous vu, on ne peut plus simplement l'opposer à l'analogique et lui attribuer des caractéristiques radicalement différentes.

On a dit par exemple que le langage verbal a une syntaxe alors que l'image en manque. Mais s'agit-il d'un manque ? Si l'image n'a guère de syntaxe, c'est que dans une certaine mesure, elle n'en a pas besoin. Ce que le langage reconstruit au moyen de substantifs, de verbes, de prépositions etc., elle le montre directement ${ }^{1}$. Ce que permettent de visualiser dans l'imaginaire mental des expressions telles que "au-dessus", "en-dessous", l'image le montre. Des connecteurs logiques tels que "ou bien...ou bien", "si...alors" ne font peutêtre que reconstruire des opérations imaginaires de comparaison et de substitution de modèles mentaux alternatifs ${ }^{2}$. Faut-il dire que l'image a de la peine à exprimer la logique ( ce qu'on admet généralement) ou à l'inverse que c'est la logique qui tente de formaliser des opérations imaginaires dont certaines pourraient bien lui échapper ?3. Laissons cette question en suspens mais admettons qu'elle concerne un problème non résolu d'avance.

1 P. Lévy écrit à propos du cinéma : "Plutôt que de se contenter d'affirmer que le cinéma n'est pas une langue parce qu'il ne possède pas de syntaxe, on pourrait tout aussi bien faire observer que, si le cinéma n'a pas besoin de grammaire, c'est parce que la fonction de la grammaire, qui est d'articuler des propositions, est toujours déjà parfaitement remplie par le langage cinématographique, et cela sans qu'il ait besoin de faire appel à l'appareillage auxiliaire d'un code", L'idéographie dynamique, op.cit., p. 57.

2 Les travaux du psycholinguiste Johnson-Laird autorisent des hypothèses dans ce sens ; cf. L'ordinateur et l'esprit, op. cit.

3 P. Lévy va résolument dans ce second sens... 
Mais si le langage sert l'imagerie mentale que, dans une certaine mesure, il sert à traduire et communiquer, il faut aussi reconnaître qu'il la transforme et tenter de préciser les modalités de cette transformation. Nous avons déjà envisagé la distance qu'introduit le langage verbal à l'égard du monde perçu comme à l'égard de sa représentation interne.

Une sémiotique cognitive aura aussi à explorer davantage les transformations introduites par les mots dans l'imagerie elle-même et à reconsidérer de ce point de vue la découverte saussurienne du principe de la différence, non pas, comme l'affirmait le dogme structuraliste, comme le fondement exclusif du sens, mais comme source des discriminations instaurées dans la positivité de l'imagerie (les catégories, les domaines, etc.) et des possibilités cognitives qui en découlent.

"La pensée, chaotique de sa nature, est forcée de se préciser en se décomposant (...)"1 disait de Saussure, ce que Bronckart reformula ainsi :

Avant l'émergence du langage, il existe certes un fonctionnement psychique pratique, mais celui-ci repose sur des formes représentatives, non seulement idiosyncrasiques, mais qui surtout constituent une masse continue et inorganisée, un amalgame d'images sans frontières nettes. Avec l'intériorisation de signifiants discontinus, des portions de formes représentatives se trouvent réorganisées en signifiés, (...), et elles sont par ce fait même érigées en véritables unités représentatives, délimitées et relativement stables. Et cette discrétisation du fonctionnement psychique constitue la condition ultime de l'émergence d'une pensée consciente ${ }^{2}$.

On ne peut mieux exprimer l'idée qui doit sous-tendre l'investigation : il n'y a pas deux modes indépendants de représentation du monde, l'analogique et le digital (ou la représentation propositionnelle et l'image); il n'y en a qu'un seul, l'iconique, mais dont le couplage avec un système extérieur de signifiants démultiplie les possibilités de différenciation, d'abstraction, etc.

1 F. DE SAUSSURE, Cours de linguistique générale, Paris, Payot, 1916, p. 156.

2 J.-P. BRONCKART, Activité langagière, textes et discours - Pour un interactionnisme socio-discursif, Neuchâtel, Delachaux et Niestlé, 1996, p. 59. 
Il s'agit donc de préciser comment les signes externes élaborés dans l'activité socio-discursive rétroagissent sur les formes de l'imagerie mentale, leur différenciation et leur organisation.

Que penser maintenant des images externes : dessins, tableaux, films, diagrammes... ? Le problème et la démarche sont semblables, même s'il ne s'agit plus, les concernant, de couplage entre deux éléments hétérogènes. Comment ces images externes interagissentelles avec l'imagerie interne?

Les images externes ont un double statut. D'une part, il ne fait pas de doute qu'elles proviennent de l'imagerie interne dont elles sont une sorte d'extériorisation. D'autre part, elles sont aussi, comme les objets de la perception, des objets externes faisant l'objet d'une réappropriation mentale (interne). Considérons successivement ces deux aspects antagonistes.

Que l'imagerie externe provienne de l'imagerie interne explique la variété de ses manifestations et, partant, la diversité des catégories discernables d'images. Les images figuratives simples (les dessins) procèdent des images mentales au sens restreint (Piaget établissait clairement le rapport entre les deux). Même les images photographiques - purs enregistrements du réel, disait Barthes, dans sa "Rhétorique de l'image"- passent par une "mentalisation" du perçu car elle est toujours le résultat d'un ajustement focal comportant point de vue, sélection, et mise en saillance de certains aspects; chaque image est la fixation d'une mise en profil ${ }^{1}$.

Les images animées proviennent et attestent de notre capacité à effectuer des transformations sur notre imagerie mentale. De même que l'image photographique n'est pas simple enregistrement du perçu, l'image cinématographique n'est pas simple enregistrement du mouvement réel. Ce dont témoignent plutôt les diverses sortes de changements visibles dans les images cinématographiques, c'est précisément de notre capacité à imaginer la transformation d'une scène visuelle en une autre ou à faire varier notre point de vue sur elle. Les différents mouvements de caméra que distinguent les théoriciens du cinéma découlent précisément de cette capacité.

1 Comme l'a bien vu la sémiologie de l'image, le problème de la ressemblance ne doit pas être posé à partir du réel lui-même (inaccessible de toute façon, d'un point de vue constructiviste), mais de la perception du réel. Cf. à ce sujet la mise au point récente de Martine JOLY dans L'image et les signes, Nathan Université, 1994, pp. 72 et sv. 
Les images plus schématiques -comme les différentes sortes de représentations graphiques: diagrammes, réseaux, cartes géographiques...- sont d'évidentes projections dans l'espace extérieur de nos modèles mentaux. Elles proviennent -et témoignent- de notre aptitude à extraire des schémas des choses, scènes et relations perçues, puis des schémas de schémas, et à agencer ces éléments dans des simulations mentales du réel à différents niveaux d'abstraction. L'aptitude à extraire des représentations schématiques se manifestent dès les premières productions graphiques comme le montrent les recherches déjà citées de $B$. Darras. L'imagerie initiale porte en effet les marques évidentes de cette aptitude : les dessins d'enfants représentent des catégories cognitives (par exemple une fleur prototypique exhibant cinq pétales au sommet d'une tige) et non des choses singulières (des fleurs concrètes) ${ }^{1}$. Ces "iconotypes" pourront ensuite prendre place dans des représentations plus complexes visualisant les relations entre les catégories de choses qu'ils symbolisent; par exemple, la fleur typique, stylisée et simplifiée à l'extrême, pourra représenter l'ensemble de la végétation dans une configuration d'éléments figurant des liens d'interdépendance ou de transformation entre états de chose (le cycle des saisons, la reproduction des végétaux, etc.). On sait le raffinement que la technique peut aujourd'hui apporter à ce type de simulation graphique.

Ce que l'on vient d'évoquer de la projection de l'espace intérieur dans l'espace extérieur suggère deux grandes questions-guides pour une sémiotique cognitive.

La première concerne l'élucidation des aptitudes cognitives sousjacentes à l'élaboration des images matérielles, celles-ci étant considérées en général d'une part, ou, d'autre part, selon les réalisations spécifiques qu'autorise la technique (dessin, photographie, cinéma, infographie... $)^{2}$. C'est en considérant les métaphores dont le langage verbal porte les traces que Lakoff et Johnson ont mis au jour la métaphorisation comme forme primordiale de conceptualisation. De même, les images externes, comme les discours, sont à considérer comme des traces révélatrices d'une activité cognitive dont doit alors

1 Cf. B. DARRAs, op.cit.

2 C'est ce type de question qui est à la base de l'enquête menée par R. Langacker à partir du langage verbal et comme celui-ci, avons-nous vu, est en rapport étroit avec l'imagerie mentale, il est probable que l'on trouve, à la base des images matérielles, les mêmes aptitudes générales que celles qu'il a découvertes à la base de l'activité langagière. 
rendre compte l'approche phénoménologique. Il semble évident, d'après l'investigation sommaire que l'on vient d'effectuer, que l'on doive déceler, à l'origine des différentes productions iconiques, des aptitudes générales telles que la comparaison, la projection métaphorique, l'extraction de schéma, les différentes modalités d'ajustement focal, l'aptitude à concevoir des transformations, etc. ${ }^{1}$. L'intérêt de cette question est surtout d'ordre psychologique mais l'approche sémiotique y gagnera néanmoins de mieux pouvoir caractériser en termes cognitifs les différents types de production auxquels elle a affaire.

La seconde question-guide est en quelque sorte l'inverse de la précédente dans la mesure où elle procède du renversement de perspective qu'implique l'idée de circularité. Cette seconde question concerne la détermination de l'imagerie mentale par les images matérielles et au-delà, par ce qui influence ces dernières : la culture et la technique. En ce qui concerne les images, la circularité peut se concevoir globalement ainsi : les images mentales (issues de l'intériorisation du monde extérieur, y compris les images matérielles) et les opérations mentales effectuées sur ces images trouvent à se spécifier dans des formes extérieures (les images matérielles techniquement possibles et socialement contrôlées pour la plupart), lesquelles sont intériorisées à leur tour, déterminant ainsi dans une certaine mesure l'imagerie mentale en lui proposant des schémas très généraux concernant à la fois les objets et la manière de les voir. Par exemple, la capacité de mettre en profil certains aspects du réel perçu a pu s'être concrétisée à un certain moment de l'histoire de l'humanité2, par le cadrage de l'image matérielle, lequel, par intériorisation, a pu infléchir la forme générale de nos images mentales en leur donnant une image de cadre sur quoi les former; notre capacité à mettre en profil par sélection et isolation en serait sortie accentuée. Cet effet en retour serait très similaire, au fond, à celui que J. Goody attribue aux listes et tableaux des premières écritures, listes et tableaux dont les lignes ou les cases bien distinctes accentuent la séparation entre les objets qui s'y inscrivent, ce qui, suite à l'intériorisation de l'image du tableau, rétroagit sur la forme de nos concepts, c'est-à-dire sur la

\footnotetext{
1 Aptitudes qu'il faut situer relativement aux acquis des nombreuses recherches sémiologiques ayant pris pour référence la notion freudienne de processus primaire et mis au jour la métaphore et la métonymie comme processus signifiant typiques de l'image.

2 Que M. Schapiro situe au néolithique.
} 
structure diacritique des schémas leur correspondant. ${ }^{1}$ Nul doute que nos dictionnaires, héritiers des listes et tableaux de l'antiquité, n'influencent par leur forme la structure des définitions qui y sont consignées. L'image intériorisée du livre (tel qu'il s'est constitué au cours des siècles avec ses différentes parties : introduction, chapitres, conclusion...) sert naturellement de cadre pour la formation de nos modèles mentaux théoriques. Dans tous les cas, on a affaire à des images schématiques servant de cadre pour d'autres images. D'une manière très générale donc, la culture et la technique produisent des schémas généraux -transsubjectifs- intériorisés par les individus et infléchissant leur imagerie individuelle spécifique. Dans le cadre de cette hypothèse, une des grandes questions que posent les nouvelles technologies semble être la suivante : dans quelle mesure la forme (réticulaire) imprimée par ces dernières à l'organisation extérieure de l'information sera-t-elle intériorisée par les individus sous forme d'une nouvelle image schématique capable de reconfigurer notre imagerie mentale (nos catégories, nos modèles et les opérations sur ces modèles) ?

Le deuxième grand aspect de l'imagerie matérielle évoqué plus haut, celui par lequel elle se présente à nous comme les objets de la perception, pose une question qui se trouve précisément à l'articulation entre les deux précédentes. Cette question porte essentiellement sur les opérations cognitives que sollicite la perception des images matérielles. Elle est sans doute la plus proprement sémiotique parce que la plus proche des signes eux-mêmes et de leur organisation plus ou moins systématique. On peut donner des exemples des investigations systématiques qu'elle appelle à différents niveaux d'analyse.

Il s'agit, au niveau élémentaire de la perception des éléments composant une image fixe, de comprendre la manière dont le regard, en se livrant à une série de comparaisons entre ces différents éléments, opère des différenciations et des assimilations jusqu'à l'obtention de formes globales avec lesquelles il puisse entrer en contact mimétique ${ }^{2}$.

$\mathrm{Au}$ niveau immédiatement supérieur, il s'agit de comprendre comment ces formes se chargent de significations suite à une série de nouvelles comparaisons prenant pour source l'expérience collective et

1 Cf. op. cit.

2 On peut trouver chez Langacker une ébauche déjà bien avancée d'une telle description des opérations élémentaires accompagnant la saisie d'une forme perceptible, cf. op.cit., p. 101 et sv. 
individuelle (mémorisée sous des formes plus ou moins schématiques) et ces formes elles-mêmes ${ }^{1}$.

$\mathrm{Au}$ niveau encore supérieur où se combinent différentes images (les plans d'un film, les vignettes d'une BD, les écrans d'un hypermédia...), il s'agira de décrire comment de nouvelles comparaisons d'image à image induisent de nouvelles assimilations et différenciations conduisant à la formation d'unités plus importantes (par ex. les séquences d'un film), englobant les précédentes et les enchâssant dans des hiérarchies d'unités signifiantes.

Et ainsi de suite à des niveaux toujours supérieurs (séquences, films, genres...) $)^{2}$.

Ces questions très générales et les réponses qu'on pourra leur donner serviront aussi de cadre pour des questions très spécifiques qui concernent les discours concrets (oraux, écrits, audiovisuels ou, maintenant, multimédiatiques) qui parcourent le tissu social en y faisant circuler toutes sortes de représentations (individuelles et collectives).

Il faudra ajouter à l'analyse de ces "discours sociaux" en termes de code ou en termes d'énonciation, une analyse en termes cognitifs. Les discours sociaux sont autant des dispositifs cognitifs que des dispositifs d'énonciation. En amont de leur production on trouve des représentations du monde - des modèles mentaux plus ou moins complexes. Ces modèles ne sont pas des décalques d'un monde objectif mais des constructions impliquant une mise en profil faisant intervenir la corporéité et des traditions diverses -disons, en gros, la culture. Chaque discours porte ainsi les marques d'un travail individuel et collectif (surtout collectif et parfois individuel) d'ajustement focal, de sélection métonymique, de projection métaphorique, de schématisation, etc., aboutissant à un certain modèle mental. Ces opérations mentales sont incorporées dans les énoncés verbaux ou iconiques composant le message, à titre de résultat ou de processus à effectuer.

1 Les études portant sur la rhétorique de l'image, inaugurées autrefois par R. Barthes et fondées sur les notions de métaphore et de métonymie, pourraient éclairer ce niveau à condition d'être réinterprétées en termes d'opérations cognitives.

2 Dans cette perspective, par exemple, les différents syntagmes autrefois différenciés par Ch. Metz dans sa grande syntagmatique de la bande-image pourraient apparaître comme le résultat, figé en routines de réalisation, de l'interaction d'une technique vouée à l'enchaînement successif des images et certaines des opérations cognitives appelées par cette successivité linéaire. 
Au plan de leur réception, ces discours sont l'objet d'un travail d'élaboration, par le destinataire, aboutissant à la reconstruction d'un modèle mental plus ou moins en correspondance avec celui du ou des émetteurs. Ce travail de reconstruction se fonde sur la saisie des intentions du destinateur ${ }^{1}$, l'accomplissement des opérations mentales qui sont incorporées au discours (une comparaison entre images, par exemple, aboutissant à une projection métaphorique) et un certain travail inférentiel pouvant se situer à plusieurs niveaux.

L'examen de ces processus peut être intéressant de différents points de vue, par exemple pour la caractérisation cognitive des types de discours (narratifs, expressifs, argumentatifs...) ou pour l'étude sociologique de catégories de discours comme le discours de presse, les films documentaires, les messages de vulgarisation scientifique, etc. Il doit aussi fournir une base pour l'évaluation de messages singuliers. Dans une perspective socio-éducative, et dans une visée de démocratie cognitive, il s'agit non seulement de voir comment ça se passe mais aussi d'évaluer si cela se passe bien et si cela pourrait mieux se passer.

1 D. Sperber et D.Wilson ont montré la nécessité de prendre en compte l'intention pour la compréhension des énoncés verbaux (La pertinence, Paris, Ed. de Minuit, 1989. Il en est probablement de même pour les "énoncés iconiques". 